\title{
Availability of platelet-rich plasma for treatment of erectile dysfunction and associated costs and efficacy: A review of current publications and Canadian data
}

Deron Britt, $\mathrm{BSc}^{1}$; Udi Blankstein, $\mathrm{MD}^{2}$; Matthew Lenardis, $\mathrm{MD}^{2}$; Alexandra Millman, $\mathrm{MD}^{2}$; Ethan Grober, $\mathrm{MD}^{3}$; Yonah Krakowsky, $\mathrm{MD}^{3}$

${ }^{1}$ University of Ottawa, Faculty of Medicine, Ottawa, ON, Canada; ${ }^{2}$ Division of Urology, University of Toronto, Toronto, ON, Canada; ${ }^{3}$ Women's College Hospital, Division of Urology, University of Toronto, Toronto, ON, Canada

Cite as: Britt D, Blankstein U, Lenardis M, et al. Availability of platelet-rich plasma for treatment of erectile dysfunction and associated costs and efficacy: A review of current publications and Canadian data. Can Urol Assoc J 2020 November 17; Epub ahead of print. http://dx.doi.org/10.5489/cuaj.6947

Published online November 17, 2020

$* * *$

\section{Abstract}

Introduction: Platelet-rich plasma (PRP) is an increasingly used unconventional treatment option for erectile dysfunction (ED). The validity of PRP as a potential treatment for ED has been proposed in limited human trials. Furthermore, the costs associated with PRP for ED treatment are not readily promoted to patients. The goal of this review was to determine the efficacy and costs of PRP based on currently available literature and Canadian data.

Methods: A comprehensive literature review of available PRP studies and current published data pertaining to cost, availability, and provider clinics globally was conducted using the PubMed database. Physicians offering genital PRP in Canada were identified using internet searches and PRP provider directories. Physician qualifications, clinic locations, and cost information were obtained from provider websites and telephone calls to identified clinics.

Results: Availability of PRP injections offered for treating ED is increasing globally. There are currently no peer-reviewed publications to substantiate anecdotal evidence pertaining to the efficacy of PRP as a viable treatment option for ED patients. Our results indicate 19 providers for PRP injections in Canada, costing on average \$1777 CAD per injection. No providers were affiliated with academic institutions and providers varied in their area of clinical speciality and training. 
Conclusions: To our knowledge, there is currently no research underway investigating the clinical efficacy of PRP for ED treatment despite its broad availability and significant cost. Patients should be informed of the lack of substantiated efficacy and safety data, as the reliability of PRP treatments requires further evaluation.

\section{Introduction}

Erectile dysfunction (ED) is a common condition defined as the inability to initiate or sustain an erection and is estimated to affect over 300 million men globally by 2025.[1],[2] Estimates of ED in the general population vary greatly between $3-76.5 \%$, but it is known to be substantially more persistent in aging demographics, with prevalence greater than $50 \%$ in men aged $40-70$ years.[2],[3] In the United States, approximately 12 million men suffer from ED influenced by common comorbidities, including, diabetes mellitus (DM), hypertension and obesity.[4] Although there are discrepancies amongst studies, ED specifically after invasive urologic procedures including radical prostatectomy (RA), has been documented to be as high as $85 \%$. [4] Compounding the physical limitations of ED, high rates of emotional distress and depression have also been documented in large-scale population studies globally.[5],[6] Recently, platelet-rich plasma (PRP) intracavernosal injections have been increasing in demand as a potential treatment modality for ED patients, despite limited data to demonstrate continuous efficacy and safety in long-term trials.[7] With a growing demand for alternative treatment options for patients, including low-intensity extracorporeal shockwave lithotripsy (LI-ESWL) and stem cell treatment (SCT), there is also a corresponding lack of standardized costs to patients through marketing from clinics supplying treatment options.[7] The publications currently available, related to human clinic trials and availability of treatment, are limited in regards to total costs of treatment, providers and professional affiliation.[8] To our knowledge, there are currently no publications of Canadian data pertaining to PRP injection clinics and associated costs to patients. This study will review the current efficacy and safety surrounding PRP injections for ED patients, as well as investigate PRP injection for ED providers and treatment costs in Canada.

\section{Methods}

\section{Literature review}

Literature review searches were performed using the Pubmed database with the initial following MeSH terms: "platelet-rich plasma” AND “erectile dysfunction”. Twenty results were initially found from those $\mathrm{MeSH}$ terms and only peer reviewed publications were used in this study. Both free full texts and texts requiring access to certain journals 
were used. Following the initial MeSH search, a second screening was initiated using the Pubmed database and the MeSH terms: "platelet-rich plasma" AND "safety and efficacy". Sixty-eight results were compiled from the MeSH terms and publications were selected based upon relevance to PRP studies and chronologic order of publication.

Supplementary publications on the usage, efficacy and safety pertaining to phosphodiesterase inhibitors and their subsequent mechanistic properties referenced in this review were collected from peer reviewed publications found in the original $\mathrm{MeSH}$ search.

\section{Data analysis}

Physicians offering PRP injections for treatment of ED in Canada were identified using Internet searches and PRP provider directories. Physician qualifications, clinic locations, and cost information were obtained from provider websites and telephone calls to identified clinics.

\section{Results}

Eighteen providers were identified across Canada that offered PRP injections for treating ED. The mean cost of a single treatment was \$1777 CAD (range \$650-\$2500). All clinics recommended at least two treatments for optimal results. No information was available regarding adverse effects or contraindications to treatment. Providers were trained in family medicine $(n=10)$, naturopathic medicine $(n=2)$, emergency medicine $(n=2)$, urology $(n=1)$, internal medicine $(n=1)$, anaesthesiology $(n=1)$, and unreported $(n=1)$. Providers were located in Ontario $(n=8)$, Alberta $(n=6)$, Quebec $(n=2)$, British Columbia $(n=1)$ and Saskatchewan $(n=1)$. No providers were identified on the east coast of Canada. No PRP provider had a university/academic affiliation.

\section{Discussion}

\section{Platelet-rich plasma: A historical perspective}

Platelet-rich plasma (PRP) has been used as a treatment option in multiple medical specialities including plastic surgery, otolaryngology and orthopaedic surgery since the 1980's.[9] PRP is theorized to provide regenerative capacity to tissues through introduction of autologous plasma with augmented platelet concentrations that far surpass that of normal blood levels.[10] These levels of platelets introduce amplified quantities of growth factors that are critical to providing stimulation for tissue repair.[10] PRP related growth factors include, but are not limited to insulin growth factor-1 (IGF-1), transforming growth factor (TGF- $\beta$ ), vascular endothelial growth factor (VEGF), platelet-derived growth factor (PDGF) and fibroblast growth factor (FGF).[10],[11] These proposed regenerative qualities have made PRP a rapidly growing unconventional therapy option for tissue regeneration in the context of, but not limited to, 
musculoskeletal repair, endometrial and follicular growth, cardiovascular applications and hair loss in other medical specialties.[10],[11]

The process of PRP preparation and extraction of corresponding essential growth factors in inactive and activated forms has been detailed extensively in previous studies.[12],[13] Recently, PRP has also been used as a treatment for ED and other urologic conditions, due in part to studies showcasing positive treatment results in other subspecialties of medicine (ie. orthopaedic and cardiac surgery).[14]

The multitude of PRP-contained growth factors have been determined to play regenerative roles via angiogenesis, collagen synthesis, restoration of smooth muscle cells and a variety of other biochemical functions.[15] While this has been extensively theorized for the use in other medical specialities, it has only been studied for urological purposes in limited ways, predominately in animal models and has currently not demonstrated consistent improvement in human trials.[25]-[32],[37]

\section{Role in erectile dysfunction}

In the context of ED, PRP-associated growth factors have been most comprehensively studied to establish their effects on erectile function via neurogenic pathways. Clinically, trauma related urethral injuries and radical prostatectomy are just two mechanisms to indirectly instigate cavernous nerve injury (CNI), leading to neurogenic ED.[16] CNI precipitates a decrease in density of nitric oxide synthase (NOS) rich nerve regions, subsequently leading to numerous biochemical events initiating smooth muscle and endothelial cell death. Corresponding fibrosis of cavernous smooth muscle increases potential constriction of penile tissue, decreasing probability of induction or sustainment of erection.[16] Penile erection is mediated by nitric oxide (NO) and NOS, both of which are critical for the initiation and maintenance of erections.[17] The proposed effects of neurotrophic growth factors, like VEGF or IGF-1, on improvement of erectile function suggest their capability of increasing downregulated gene expression fundamental to improving erectile quality through these or similar pathways.[17],[18] Increasing NO, and subsequent conversion to cGMP, is vital in promoting relaxation of corpus cavernosum smooth muscle and promoting effective vasodilation to sustain penile erection.[19],[20] As an example, PDE-5 inhibitors (ie. Sildenafil or Vardenafil) limit the breakdown of cGMP, leading to prolonged elevated cGMP levels in erectile tissue and sustained penile erections. [20] These pharmaceutical agents, however, have been a proven treatment method through long-term follow-up studies, standardized dosing protocols and safety trials in large cohort studies and are presently considered effective and safe treatment agents for patients.[21]-[24] 


\section{Animal model trials}

Efficacy for ED treatment involving PRP has been primarily investigated in animal trials via cavernous nerve crush injury or cavernous neurotomy studies.[25] Multiple studies have demonstrated that PRP associated growth factors are critical for increased neuronal nitric oxide synthase (nNOS), promoting neural regeneration and potential improvement in erectile function.[25]-[30] Erectile function improvements in rat models have also been documented following PRP injections in groups with bilateral CNI.[29] Intensified TGF- $\beta 1$ levels and axon preservation was documented in these studies at four-week follow-up, along with improved erectile function in the PRP-treated group.[29] Optimized PRP, composed of increased levels of PDGF in subsequent studies was found to improve recovery as well as erectile function in groups who underwent bilateral $\mathrm{CN}$ crush injury.[30] More recent studies in rat models have found similar trends in which increased myelination and improved erectile function suggest that growth factors including, TGF- $\beta$, IGF-1 and VEGF, support regeneration of CNs and positively impact nNOS post-CNI.[25]

\section{Human clinical trials}

There have been a limited number of human clinical trials investigating PRP in patients with ED to this date. Epifanova et al., examined essential growth factors for improving erectile function in ED patients from PRP samples obtained from control $(n=12)$ and patient $(n=12)$ groups. Concentrations of growth factors from both population groups were identified through flow cytometry with results of the study indicating that the overall concentration of growth factors was higher in the control group (1480) when compared to the patient group (1232), concluding that the improvements in erectile function proposed were due to the active contents of PRP obtained (ie. FGF, PDGF and VEGF).[31]

The most recent human trials involving PRP have discussed the potential theoretical utilization for ED therapy as well as other urological illnesses.[32] However, the concerns surrounding early washout of PRP was considered in these trials, and the suggestion was made to convert PRP to platelet rich fibrin matrix (PRFM) prior to injection in patients.[32] Results of this study suggest that there were no major complications found in the patient population $(n=4)$ during follow-up and pre and postinjection International Index of Erectile Function (IIEF-5) scores did not indicate decline in the patient population.[32] These studies have given promise to PRP being utilized as a treatment option for ED.

\section{Safety of platelet-rich plasma for erectile dysfunction treatment}

Investigations surrounding the safety of PRP injections as a form of regenerative therapy has been extensively discussed and reviewed in non-urology based medical specialities in both human and animal population publications.[33] The general consensus from these 
previous analyses suggests that PRP injections have been deemed as a safe modality for treatment in multiple practices of medicine that includes orthopaedics, dermatology, musculoskeletal tissue regeneration and other applications.[33]-[35] Regarding the safety of PRP as a prospective therapy option for ED, evidence from small group animal based studies involving novel rat models with experimentally induced neurogenic penile injuries has not shown the long-term effects on treated animals, and therefore overall safety has not been verified.[29],[30]

The most recent available studies published on human clinical groups are limited in regards to both qualitative and quantitative data but do suggest that PRP injections can be a safe modality for treatment, with bruising at the site of injection being the only reported ill effect in short-term follow-up in a small percentage of test subjects.[32] However, further investigations involving larger cohort studies, random control trials and long-term follow up of patients is critical to confirm or refute these conclusions.[8]

\section{Efficacy of platelet-rich plasma for erectile dysfunction treatment}

PRP has been shown to improve erectile function in animal studies involving induced neurogenic ED in small-sample populations.[36] However, these studies have not demonstrated long-term cessation of ED in animal models and further improvement on standardized quantities of growth factors are required to substantiate efficacy of treatment. Furthermore, larger cohort groups would provide a broader population base to sample and improve validity of future results.[8],[32]

The available published trials that include human data have proposed that PRP could be provided as a plausible ED treatment option for patients from initial results demonstrating both their safety and efficacy post-injection.[31],[32] However, systematic analysis revealed extensive limitations in these studies due to small group sizes, short follow-up periods, the absence of control and placebo groups and missing quality and quantity analysis of PRP.[31],[32],[36],[37] As such, review of the current published animal and human data suggests there is currently no concrete evidence supporting the validity of PRP as a reliable technique for the treatment of ED. Similarly, available data pertaining to the efficacy of PRP injections in other medical subspecialties as a viable treatment modality is reflected as predominately anecdotal and found to be lacking both qualitative and quantitative corroborating evidence.[38]-[40] Methods required for standardization of PRP processing and associated concentrations of growth factors, as well as more qualitative randomized control trials involving larger patient cohorts and subsequent long-term follow-up investigations are necessary to properly assess efficacy and safety of treatment in patient populations diagnosed with ED and other urologic conditions.[8],[41],[42]

\section{Availability of platelet-rich plasma in North America and internationally}


Novel techniques and treatments for ED, including PRP, LI-ESWL and SCT, are increasing in both availability and demand throughout Canada and internationally.[1],[7],[8] Scott et al., found that global trends in PRP associated treatments for ED are steadily increasing in demand, primarily in Australia, New Zealand and the United States.[7],[8] The study systematically evaluated the marketing of PRP through various global providers and any trends associated with PRP and ED, including the availability of advertised products.[7] Results of this analysis determined that the most accessible marketed forms of PRP injections for ED, including the Priapus shot or P-shot, had 683 providers registered in 19 countries at the time of their data acquisition.[7] The majority of providers identified did not provide the costs of intracavernous injections of PRP marketed online to prospective patients and most providers of PRP were not certified urologists in any of the countries offering these injections.[8] Given the increase in marketing of PRP for ED, as well as the increased interest from the public globally, greater transparency in regards to the efficacy and cost of injections is warranted and larger scientific studies on patient populations are necessary to establish the current efficacy and safety of these procedures.[8]

\section{Canadian data}

In regards to cost, availability and clinical provider affiliations and trends associated with PRP injections in Canada, the data obtained in our study coincides with similar findings globally.[7],[8] Currently, the average cost per injection in Canada is estimated to be \$1777 CDN, similar to Australia (\$1500 AUD) and the United States (\$1500 - \$2200 USD).[8] Out of the eighteen PRP providers in Canada for the treatment of ED, a substantial portion $(n=5)$ did not provide details regarding cost of treatment via promotional websites, coinciding with international marketing tendencies.[8]

Furthermore, all Canadian clinics $(n=18)$ emphasized a minimum of two treatments to optimize results for men being treated for erectile dysfunction. Expenses affiliated with follow-up injections increased in cost or are not detailed, which follows a trend in previous publications declaring that multiple providers promote serial injections of PRP to maximize treatment efficacy.[8] With the most recent published international data suggesting that PRP injections can cost thousands of dollars per treatment, and with no immediate government subsidization offered to patients or minimal regulation of unsubstantiated therapies, it is critical for patients to understand the current validity and safety associated with these methods.[7],[8] Another revelation of the Canadian data that warrants further investigation was that none of the providers of PRP injections were found to be affiliated with any academic institutions. Future insight as to why this may be could be of great value for future studies to explore. Patient understanding of the current information surrounding these methods can be improved in the future by educating patients in regards to the recent published efficacy and safety data pertaining to PRP, as well as the current peer reviewed evidence either supporting or contrary to advertised 
treatments from clinical providers. Furthermore, transparency from clinics offering PRP treatment regarding the state of the evidence is vital for building patient rapport and obtaining informed consent. The costs associated with PRP injections, and potential follow-up or ancillary fees, are extensive and can place added financial, emotional or physical stress on the patient, especially if PRP is unsuccessful.

\section{Conclusions}

PRP injections are an ever-increasing method advertised as a viable treatment for a variety of urologic conditions including male and female dysfunction, and more specifically, ED in the male population. PRP is available in most parts of Canada at a significant cost, with no university-affiliated providers currently offering PRP at the time of this review. To our knowledge, there is currently no research underway investigating its clinical efficacy to endorse its clinical use and corroborate limited human trials.

Review of the current literature suggests that there is currently not enough substantiated data to warrant PRP being a practical treatment option for patients suffering from ED. More extensive human trials, that include standardized control groups and protocols along with long-term follow up data are necessary to provide further insight to if PRP can be incorporated as a routine option for treatment of ED.

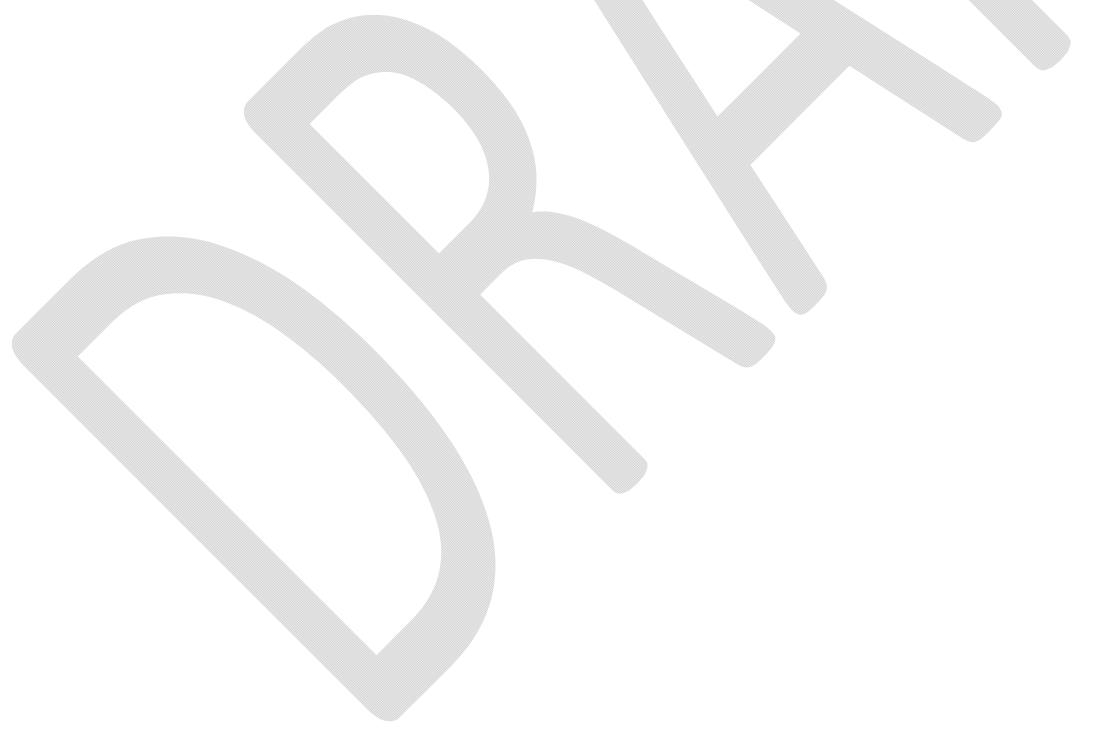




\section{References}

1. Campbell JD, Milenkovic U, Usta MF, et al. The good, bad, and the ugly of regenerative therapies for erectile dysfunction. Transl Androl Urol 2020;9:S252S261.

2. Raheem AA, Kell P. Patient preference and satisfaction in erectile dysfunction therapy: A comparison of the three phosphodiesterase-5 inhibitors sildenafil, vardenafil and tadalafil. Patient Prefer Adherence 2009;3:99-104.

3. Kessler A, Sollie S, Challacombe B, et al. The global prevalence of erectile dysfunction: A review. BJU international 2019;124:587-99.

4. Rew KT, Heidelbaugh JJ. Erectile Dysfunction. Am Fam Physician 2016;94:82027.

5. Shiri R, Koskimäki J, Tammela TL, et al. Bidirectional relationship between depression and erectile dysfunction. J Urol 2007;177:669-73.

6. Nelson CJ, Mulhall JP, Roth AJ. The association between erectile dysfunction and depressive symptoms in men treated for prostate cancer. J Sex Med 2011;8:56066.

7. Scott S, McKnoulty M, Roberts M, et al. Efficacy, commercialisation and evidence relating to platelet rich plasma injections for the treatment of erectile dysfunction. Bju International 2018;121:58.

8. Scott S, Roberts M, Chung E. Platelet-rich plasma and treatment of erectile dysfunction: Critical review of literature and global trends in platelet-rich plasma clinics. Sex Med Rev 2019;7:306-12.

9. Hall MP, Band PA, Meislin RJ, et al. Platelet-rich plasma: Current concepts and application in sports medicine. JAAOS-Journal of the American Academy of Orthopaedic Surgeons 2009; 17:602-08.

10. Wu PI, Diaz R, Borg-Stein J. Platelet-rich plasma. Phys Med Rehabil Clin N Am 2016;27:825-53.

11. Bos-Mikich A, de Oliveira R, Frantz N. Platelet-rich plasma therapy and reproductive medicine. J Assist Reprod Genet 2018;35:753-56.

12. Kushida S, Kakudo N, Morimoto N, et al. Platelet and growth factor concentrations in activated platelet-rich plasma: A comparison of seven commercial separation systems. J Artif Organs 2014;17:186-92.

13. Sumida E, Iwasaki Y, Akiyoshi K, et al. Platelet separation from whole blood in an aqueous two-phase system with water-soluble polymers. J Pharmacol Sci 2006;101:91-7.

14. Patel AN, Selzman CH, Kumpati GS, et al. Evaluation of autologous platelet rich plasma for cardiac surgery: Outcome analysis of 2000 patients. J Cardiothorac Surg 2016;11:62.

15. Dhurat R, Sukesh M. Principles and methods of preparation of platelet-rich plasma: A review and author's perspective. J Cutan Aesthet Surg 2014;7:189-97.

16. Gu, X, Shi H, Matz E, et al. Long-term therapeutic effect of cell therapy on improvement in erectile function in a rat model with pelvic neurovascular injury. BJU international 2019;124:145-54. 
17. Campbell JD, Burnett AL. Neuroprotective and nerve regenerative approaches for treatment of erectile dysfunction after cavernous nerve injury. Int J Mol Sci 2017;18:1794.

18. Musicki B, Palese MA, Crone JK, et al. Phosphorylated endothelial nitric oxide synthase mediates vascular endothelial growth factor-induced penile erection. Biol Reprod 2004;70:282-9.

19. Bivalacqua TJ, Usta MF, Champion HC, et al. Endothelial dysfunction in erectile dysfunction: Role of the endothelium in erectile physiology and disease. J. Androl 2003;24:S17-S37.

20. Burnett A.L. Role of nitric oxide in the physiology of erection. Biol. Reprod 1995;52:485-89.

21. Yafi FA, Sharlip ID, Becher EF. Update on the safety of phosphodiesterase type 5 inhibitors for the treatment of erectile dysfunction. Sex Med Rev 2018;6:242-52.

22. Valiquette L, Montorsi F, Auerbach S. Vardenafil demonstrates first-dose success and reliability of penetration and maintenance of erection in men with erectile dysfunction. Can Urol Assoc J 2008;2:187-95.

23. Ljunggren $\mathrm{C}$, Hedelin $\mathrm{H}$, Salomonsson K, et al. Giving patients with erectile dysfunction the opportunity to try all three available phosphodiesterase type 5 inhibitors contributes to better long-term treatment compliance. J Sex Med 2008;5:469-75.

24. Ströberg P, Hedelin H, Ljunggren C. Prescribing all phosphodiesterase 5 inhibitors to a patient with erectile dysfunction--a realistic and feasible option in everyday clinical practice--outcomes of a simple treatment regime. Eur Urol 2006;49:900-07.

25. Ding XG, Li SW, Zheng XM, et al. The effect of platelet- rich plasma on cavernous nerve regeneration in a rat model. Asian J Androl 2009;11:215-21.

26. Lin CS, Ho HC, Chen KC, et al. Intra- cavernosal injection of vascular endothelial growth factor induces nitric oxide synthase isoforms. BJU Int 2002; 89:955-60.

27. Chen KC, Minor TX, Rahman NU, et al. The additive erectile recovery effect of brain-derived neurotrophic factor combined with vascular endothelial growth factor in a rat model of neurogenic impotence. BJU Int 2005;95:1077-80.

28. Hsieh PS, Bochinski DJ, Lin GT, et al. The effect of vascular endothelial growth factor and brain- derived neurotrophic factor on cavernosal nerve regeneration in a nerve-crush rat model. BJU Int 2003;92:470-5.

29. Wu CC, Wu YN, Ho HO, et al. The neuroprotective effect of platelet-rich plasma on erectile function in bilateral cavernous nerve injury rat model. J Sex Med 2012;9:2838-48.

30. Wu YN, Wu CC, Sheu MT, et al. Optimization of platelet-rich plasma and its effects on the recovery of erectile function after bilateral cavernous nerve injury in a rat model. J Tissue Eng Regen Med 2016;10:294-304.

31. Epifanova MV, Chalyi ME, Krasnov AO. Investigation of mechanisms of action of growth factors of autologous platelet-rich plasma used to treat erectile dysfunction. Urologiia 2017;(4):46-8. 
32. Matz EL, Pearlman AM, Terlecki RP. Safety and feasibility of platelet rich fibrin matrix injections for treatment of common urologic conditions. Invest. Clin. Urol 2018;59:61-5.

33. Sampson S, Gerhardt M, Mandelbaum B. Platelet rich plasma injection grafts for musculoskeletal injuries: a review. Current reviews in musculoskeletal medicine 2008;1:65-174.

34. Fukawa T, Yamaguchi S, Akatsu Y, et al. Safety and efficacy of intra-articular injection of platelet-rich plasma in patients with ankle osteoarthritis. Foot Ankle Int 2017;38:596-604.

35. Suthar M, Gupta S, Bukhari S, et al. Treatment of chronic non-healing ulcers using autologous platelet rich plasma: A case series. J Biomed Sci 2017;24:16.

36. Krzastek SC, Bopp J, Smith RP, et al. Recent advances in the understanding and management of erectile dysfunction. F1000Res 2019;8:102.

37. Epifanova MV, Gvasalia BR, Durashov MA, et al. Platelet- rich plasma therapy for male sexual dysfunction: Myth or reality? Sex Med Rev 2020;8:106-13.

38. Ayatollahi A, Hosseini H, Gholami J, et al. Platelet rich plasma for treatment of non-scarring hair loss: Systematic review of literature. J Dermatolog Treat 2017;28:574-81.

39. Urits I, Viswanath O, Galasso AC, et al. Platelet-rich plasma for the treatment of low back pain: A comprehensive review. Curr Pain Headache Rep 2019;23:52.

40. Hamilton BH, Best TM. Platelet-enriched plasma and muscle strain injuries: Challenges imposed by the burden of proof. Clin J Sport Med 2011;21:31-6.

41. Chung E. A review of current and emerging therapeutic options for erectile dysfunction. Med Sci (Basel) 2019;7:91.

42. Chahla J, Cinque ME, Piuzzi NS, et al. A call for standardization in platelet-rich plasma preparation protocols and composition reporting: A systematic review of the clinical orthopaedic literature. J. Bone Joint Surg Am 2017;99:1769-79.

Competing interests: Dr. Krakowsky has been a consultant for Paladin, Verity and Pfizer. Dr. Grober has been an investor for NHB Labs, a research supporter for Boston Scientific and a consultant for Paladin. All other authors affiliated with this paper report no competing financial or personal interests. 